\title{
How to assess and manage hypertension during and after pregnancy
}

Louise A McKenna', Shahyza S Huda ${ }^{1}$, Dilys J Freeman ${ }^{1}$ \& Eleanor Jarvie*1

\section{Practice Points}

- UK maternal deaths secondary to hypertensive disorders of pregnancy have been attributed to substandard care.

- Accurate diagnosis of hypertension depends on precise blood pressure measurements.

- The main types of hypertensive disorders of pregnancy are: chronic, gestational and pre-eclampsia (de novo or superimposed).

- All pregnant women with hypertension should be closely monitored for the development of pre-eclampsia.

- Delivery before 34 weeks' gestation should be offered if severe, refractory hypertension develops or documented threshold intervention values are breached.

- Birth should take place following anesthetic and neonatal consultation, and should occur following maternal corticosteroid administration.

- Vigilant blood pressure monitoring and antihypertensive regimen adherence is essential postdelivery.

- Hypertensive control should be achieved prior to discharge.

- Hypertensive disorders in pregnancy increase the risk of maternal cardiovascular and renal disease in later life. All clinicians should inform their patients of these increased risks to ensure regular, routine monitoring.

Future $\because$ Medicine 


\section{SUMMARY Hypertensive disorders of pregnancy are increasingly important complications} of which clinicians should have an up-to-date knowledge to facilitate prompt recognition, diagnosis and management. These disorders affect a growing number of pregnancies worldwide, with incidence rates likely to increase in the future commensurate with increasing maternal age and maternal comorbidities independent of age, with consequent effects on maternal and fetal/neonatal morbidity and mortality rates. This article mainly focuses on management within the UK of these disorders, examining their current working definitions, detection methods and recent developments in screening tool development. The current NICE-recommended strategies for treating these disorders and minimizing their occurrence in pregnancy are also explored. In addition, the association between adverse pregnancy outcome and increased risk of future maternal and offspring cardiovascular disease is described, with comments on future strategies to help minimize these potential risks.

Hypertensive disorders of pregnancy, which encompass pre-existing chronic hypertension, gestational hypertension and pre-eclampsia, are the most commonly encountered medical complications of pregnancy worldwide. These disorders are estimated to affect a third of pregnancies in the UK and in the developing world, and are known to be significant contributors to annual rates of maternal and perinatal morbidity and mortality worldwide $[1,2]$. The Eighth Report on Confidential Enquiries into Maternal Deaths in the UK produced by the Centre for Maternal and Child Enquiries reviewed maternal deaths recorded in the UK between 2006 and 2008. It identified hypertensive disorders (pre-eclampsia and eclampsia) as the second most common direct cause of maternal deaths, with related mortality rates remaining relatively static $(0.83$ deaths per 100,000 maternities [95\% CI: 0.53-1.30] between 2006 and 2008 compared with 0.85 deaths per 100,000 maternities [95\% CI: 0.54-1.35] between 2003 and 2005) [3]. Although the maternal mortality rate may have remained static, there is considerable morbidity associated with hypertensive disorders in pregnancy as a result of increasing rates of maternal comorbidities (e.g., obesity, diabetes and cardiovascular disease, among others) in the increasingly older pregnant population. Alarmingly, of the 22 UK maternal deaths that occurred between 2006 and 2008 directly related to pre-eclampsia, eclampsia and acute fatty liver of pregnancy, 20 were attributed to substandard clinical care, highlighting the urgent need to review and update the current definitions and clinical management of these disorders, to help reduce their potential catastrophic impact on the lives of mothers and babies affected both during pregnancy and in later life.

Healthy pregnancy \& physiological changes to blood pressure

From at least 6 weeks' gestation, maternal blood pressure (BP) has been found to progressively fall due to a reduction in systemic vascular resistance, thought to be secondary to vessel remodeling by maternal hormones [4]. From approximately 20 weeks' gestation, both systolic and diastolic BP progressively increase towards term [5]. BP has been found to track moderately during pregnancy (i.e., women who started in the highest BP tertile in the first trimester remained in the highest tertile in the third trimester) [6], which has been recognized to be influenced by individual maternal characteristics, in particular, gestational weight gain, although research is required to further explore this relationship [7]. Postnatally, BP drops in the $48 \mathrm{~h}$ after birth, before rising back up to nonpregnant levels [8].

Definition of arterial hypertension in pregnancy

Hypertension in pregnancy is diagnosed as either an absolute BP rise or a relative rise above the recorded booking appointment BP [1]. The accurate diagnosis of maternal hypertension depends on precise BP measurements (i.e., with attention to cuff size, position of the arm at heart level and calibration of equipment) [1]. Conventionally, a systolic BP over $140 \mathrm{mmHg}$ 
or a diastolic BP over $90 \mathrm{mmHg}$ is defined as hypertension in the nonpregnant adult [1]. However, the physiological BP changes that occur as the maternal cardiovascular system adapts to the growing fetus means it is difficult to use specific diagnostic BP values as standard across gestation [5].

\section{BP measurement in pregnancy}

To diagnose maternal hypertension, a BP reading should be elevated on two separate occasions, measured at least $4 \mathrm{~h}$ apart, using an appropriately sized BP cuff as the woman sits with her arm at heart level [1]. From the second trimester onwards, BP can be measured sitting or in the lateral supine position to avoid reduced maternal venous return by the gravid uterus [1]. The Korotkoff sounds used to measure BP should be I and V (disappearance) as opposed to IV (muffling), as these are more reproducible [9] and more accurate in determining maternal diastolic BP [10]. The widespread use of automated BP apparatus in modern obstetrics is controversial as this method has been found to systematically underestimate systolic and diastolic BP in both healthy and pre-eclamptic pregnancies by $10-20 \mathrm{mmHg}$ [11]. Therefore, the consideration of invasive in-patient monitoring may be warranted in severe cases of pre-eclampsia [11]. In obstetric practice, 24-h ambulatory BP monitoring is common, but its use has not been fully evaluated for maternal hypertensive disorders. Monitoring ambulatory BP may minimize the prescription of antihypertensives for 'white-coat hypertension' in the clinical setting (estimated at $30 \%$ of pregnant women [12]), as well as identifying those women with elevated BP outside the clinical setting.

Classification of hypertensive disorders of pregnancy

Several different classification systems of hypertensive disorders of pregnancy exist, which leads to difficulty in comparing worldwide data on this condition. The most widely used definition is that of the International Society for the Study of Hypertension in Pregnancy which is, in particular, used for research and epidemiological purposes (Table 1) [13]. Briefly, the four disorders classified are: chronic hypertension, gestational hypertension, de novo pre-eclampsia (and its associated disorders) and chronic hypertension with superimposed pre-eclampsia. Clinically, these definitions are less important since all women with raised BP should be carefully monitored throughout pregnancy, especially for the associated features of developing pre-eclampsia.

The emphasis on the relative values of diastolic and systolic BP in defining hypertension has shifted. Classically, a severely raised diastolic BP $(>110 \mathrm{mmHg}$ ) was considered more predictive of stroke risk. However, more recent evidence from an obstetric case series demonstrated that this did not predate stroke, whereas systolic BP did [14]. The importance of controlling maternal systolic BP reflects the evidence in the adult nonpregnant population where a better correlation is noted between the degree of systolic BP elevation, as opposed to diastolic BP, and the risk of hemorrhagic stroke (the most common type of stroke affecting the obstetric population) [15].

Current evidence suggests that the pattern of gestational BP change may distinguish women with essential hypertension, gestational hypertension and pre-eclampsia from each other and from normotensive women [16]. Even in early pregnancy, these distinct patterns may be useful for identifying women at risk of developing a hypertensive disorder later in pregnancy [16]. A recent study analyzed the antenatal clinic BP measurements (median: 14 per woman) of 13,016 women from the Avon Longitudinal study of Parents and Children at different gestational time points (8-18, 18-30, 30-36 and $\geq 36$ weeks' gestation) and found that women who had essential hypertension and those who developed gestational hypertension or pre-eclampsia had higher BP at 8 weeks' gestation (baseline) than normotensive women [16]. Maternal BP increased more rapidly from 18 weeks onward in gestational hypertensive and pre-eclamptic pregnancies, and from 30 weeks onward in essential hypertensive, compared with normotensive pregnancies [16]. A more rapid rise in $\mathrm{BP}$ occurring beyond 30 weeks of pregnancy was a risk factor for the development of pre-eclampsia [16]. This study suggested the preliminary hypothesis of a distinctive clinical phenotype, apparent prior to the clinical manifestation of these disorders, which may be used to identify those asymptomatic women at increased risk early.

\section{Chronic hypertension}

Chronic hypertension is defined as an elevated $\mathrm{BP}$ recorded at the time of booking appointment 


\begin{tabular}{|c|c|c|}
\hline \multicolumn{2}{|l|}{ Term } & \multirow{2}{*}{$\begin{array}{l}\text { Definition } \\
140-149 / 90-99 \mathrm{mmHg}\end{array}$} \\
\hline Hypertension & Mild & \\
\hline & Moderate & $150-159 / 100-109 \mathrm{mmHg}$ \\
\hline & Severe & $\geq 160 / \geq 110 \mathrm{mmHg}$ \\
\hline \multicolumn{2}{|l|}{ Chronic } & $\begin{array}{l}\text { Hypertension present at the booking visit, or before } 20 \text { weeks' } \\
\text { gestation }\end{array}$ \\
\hline \multicolumn{2}{|l|}{ Gestational } & $\begin{array}{l}\text { New hypertension presenting after } 20 \text { weeks without significant } \\
\text { proteinuria }\end{array}$ \\
\hline \multicolumn{2}{|l|}{ Pre-eclampsia } & $\begin{array}{l}\text { New hypertension presenting after } 20 \text { weeks' gestation with } \\
\text { proteinuria of } \geq 0.3 \mathrm{~g} / 24 \mathrm{~h} \text {, or } \geq 2+\text { on dipstick testing (in the absence } \\
\text { of renal disease or infection) }\end{array}$ \\
\hline \multicolumn{2}{|c|}{ Severe pre-eclampsia } & $\begin{array}{l}\text { Pre-eclampsia with severe hypertension and/or with symptoms, } \\
\text { and/or biochemical and/or hematological impairment }\end{array}$ \\
\hline \multicolumn{2}{|l|}{ Eclampsia } & A convulsive condition associated with pre-eclampsia \\
\hline
\end{tabular}

or before 20 weeks' gestation, and is either of primary (essential) or secondary etiology [1]. A prepregnancy BP is rarely documented in young, healthy women, therefore, the diagnosis of chronic hypertension may be difficult to make clinically if the first presentation is during pregnancy. If there is no prior history, or it has not previously been documented, chronic hypertension is diagnosed at the time of pregnancy by a persistent BP elevation $\geq 140 / 90 \mathrm{mmHg}$ prior to 20 weeks' gestation or if maternal BP is elevated 6 weeks postdelivery [17]. Misdiagnosis may occur later in pregnancy, due to the physiological fall in BP prior to 20 weeks' gestation masking pre-existing chronic hypertension [4]. It is particularly important to exclude the rare diagnosis of pre-eclampsia in the second trimester.

Chronic hypertension is common in women at the extremes of child-bearing age and its prevalence is set to increase with rising maternal age at presentation and increasing worldwide prevalence of cardiovascular disease in the adult female population [101]. The Eighth Report on Confidential Enquiries into Maternal Deaths in the UK published in 2011 confirmed the increasing incidence of older mothers and highlighted their increased risk of pregnancy complications such as pre-eclampsia, gestational diabetes and placental abruption [3], likely to be due to increasing rates of metabolic and cardiovascular disease [101]. The rising incidence of maternal obesity was also noted in this report [3] and will likely contribute to the increasing rates of hypertensive disease, especially preeclampsia, whose incidence risk doubles for every $5-7 \mathrm{~kg} / \mathrm{m}^{2}$ increase in prepregnancy BMI [18].
A secondary etiology is identified in the majority of cases, with underlying causes including: cardiovascular, renal, endocrine and connective tissue disease (Box 1) [19]. An assessment of gestational hypertensive disease is made on the presence of any of these secondary causes or known target organ damage, in combination with past obstetric history and the severity of hypertension. Chronic hypertension is sometimes classified in the literature as mild, moderate or severe (Table 1), reflecting the increased risks associated with a greater degree of hypertension. Current evidence suggests that mild/moderate disease is associated with increased risk of placental abruption and fetal intrauterine growth restriction [20], although direct causality has not been established. Severe chronic hypertension prior to 20 weeks' gestation has been reported, in one study, to be linked with a $46 \%$ increased risk of developing superimposed pre-eclampsia [20], with its own additional increased risks of maternal and fetal complications. Superimposed pre-eclampsia is diagnosed in females with chronic hypertension or renal disease if they develop new-onset proteinuria (or a sudden increase in preexisting proteinuria levels), or a sudden increase in hypertension or hemolysis, elevated liver enzymes and low platelet count syndrome [20].

\section{Gestational hypertension}

Gestational hypertension is estimated to complicate $6-7 \%$ of pregnancies in the second half of pregnancy [21]. The risk of superimposed pre-eclampsia developing is influenced by the gestation at which hypertension first develops. A diagnosis after 36 weeks of pregnancy is 
associated with a $10 \%$ risk of developing preeclampsia [21]. An extremely uncommon cause of rapid hypertension is a pheochromocytoma. Although this is a rare cause, this diagnosis should be considered in a woman with the clinical symptoms of excessive catecholamine excretion (e.g., tachycardia, diaphoresis and sudden-onset headache) who is not responding to pharmacological treatment [22].

\section{Pre-eclampsia}

Pre-eclampsia is a multisystem disorder of pregnancy characterized by generalized endothelial dysfunction, with differential organ targeting [23]. In the UK, the NICE Hypertension in Pregnancy guidelines (revised January 2011) define pre-eclampsia as new hypertension (a systolic BP $\geq 140 \mathrm{mmHg}$, diastolic BP $\geq 90 \mathrm{mmHg}$, on repeated readings) presenting after 20 weeks' gestation with significant proteinuria $(\geq 0.3 \mathrm{~g} / 24 \mathrm{~h}$ or $2+$ on dipstick testing), in the absence of renal disease or infection [102]. Both BP and proteinuria criteria should be met within no more than 1 week of each other [17]. Eclampsia is defined as a generalized tonic-clonic seizure occurring on the background of known pre-eclampsia or as the presenting symptom [102].

It is estimated that pre-eclampsia complicates $2-8 \%$ of UK pregnancies [23], affecting approximately 30,000 women annually [23]. The outcomes of these pregnancies are determined largely by gestational age at onset, quality of clinical management and the presence of preexisting medical disorders, with several other risk factors also identified (Figure 1). Adverse maternal and perinatal outcomes are not universal, indicating the wide clinical spectrum of this disorder. Consequently, it is often subclassified in the literature as either mild or severe disease with early or late gestational onset. However, these definitions can prove misleading with 'mild' pre-eclampsia developing into eclampsia, bypassing any severe disease signs and, therefore, should be used with caution in the clinical setting. The NICE guidance defines severe pre-eclampsia as either the presence of severe hypertension (systolic BP $\geq 160 \mathrm{mmHg}$, diastolic $\mathrm{BP} \geq 110 \mathrm{mmHg}$ ) or mild/moderate hypertension with severe proteinuria $(>5 \mathrm{~g} / 24 \mathrm{~h}$ ) [102]. Pre-eclampsia is also considered severe if there is the presence of additional systemic complications, including: pulmonary edema, seizures, oliguria $(<500 \mathrm{ml} / 24 \mathrm{~h})$, thrombocytopenia (platelets $<100,000 / \mu 1$ ), abnormal liver enzymes in association with persistent epigastric or right upper quadrant pain and persistent or severe CNS symptoms (e.g., altered mental status, headaches or blurred vision or blindness) [102]. Pre-eclampsia is defined as either early- ( $\leq 34$ weeks' gestation) or lateonset although it is still unclear as to why some women develop this disorder with varied clinical presentations and outcomes. Current evidence indicates that early- and late-onset pre-eclampsia, with or without associated fetal growth restriction, are two different forms of the condition and, thus, are likely to have different etiologies [22]. Early-onset pre-eclampsia is likely to be due to an initial failure of placentation, whereas late-onset pre-eclampsia may represent an abnormality in the maternal physiological response to pregnancy [23]. The detection and management of early-onset pre-eclampsia, with its significantly higher maternal and perinatal

Box 1. Causes of secondary chronic
hypertension in pregnancy.
Cardiovascular
" Coarctation of aorta
" Vasculitis
Renal
- Renovascular disease
" Pre-existing renal disease
- Diabetic nephropathy
- Chronic glomerulonephritis
- Tubulointerstitial nephritis
- Chronic pyelonephritis
- Polycystic kidney disease
Endocrine
- Obesity
- Diabetes mellitus
" Hyperthyroidism
- Primary hyperaldosteronism
" Cushing's syndrome
" Pheochromocytoma
Connective tissue disease
" Systemic lupus erythematosus
- Systemic sclerosis
- Others




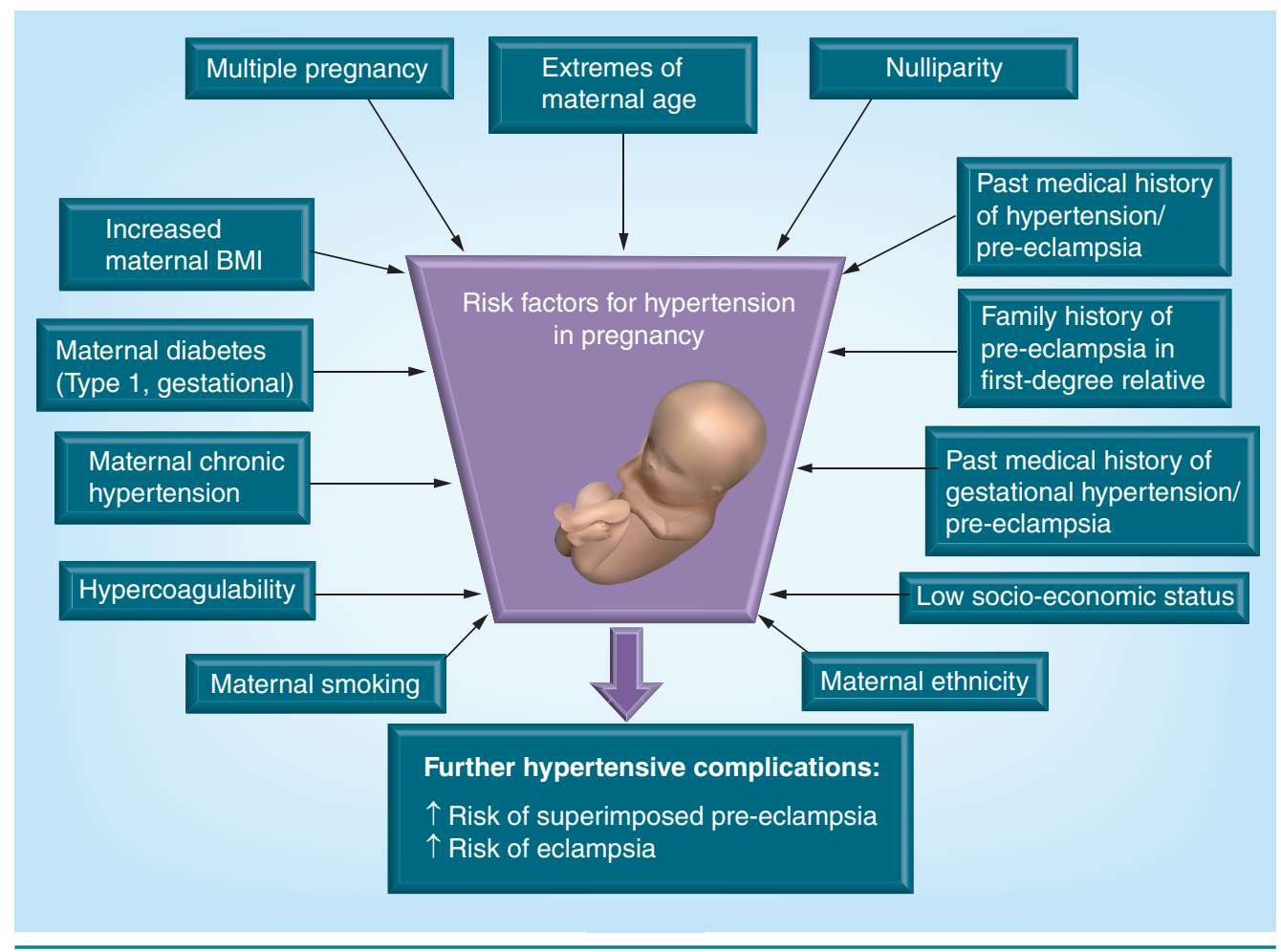

Figure 1. Recognizable clinical risk factors for hypertension in pregnancy.

Data taken from $[1,17,21]$.

morbidity and mortality, is perhaps deemed to be of greatest importance. Annual maternal mortality is estimated at approximately 50,000 women worldwide [24] and is often attributable to multisystem complications, such as placental abruption, disseminated intravascular coagulopathy, hemolysis, elevated liver enzymes and low platelet count syndrome, lung/kidney/liver failure or liver/intracerebral hemorrhage.

Maternal cardiac disease, both acute and long term, is also a known complication of preeclampsia/eclampsia and is currently the most common cause of indirect maternal death in the UK [3]. Fetal morbidities include intrauterine growth restriction, hypoxic neurological injury and iatrogenic prematurity, as well as perinatal death or chronic medical conditions associated with low birth weight and prematurity [3].

Although major breakthroughs have been made in targeting the pathophysiology of pre-eclampsia, the initiating event remains enigmatic. Pre-eclampsia is resolved by the delivery of the placenta and a retained placenta or molar pregnancy can lead to the development of pre-eclampsia, linking the primary defect to the trophoblast. This disease is believed to represent a failure of trophoblast invasion and adequate placentation. The trophoblast implantation site is immunologically active, and it is hypothesized that trophoblasts release chemokines stimulating immune cell recruitment and induction of a type 2 immune phenotype [25]. Consequently, poor placentation may be due to dysregulation of the maternal immune system [26]. The release of placental factors into the maternal circulation, secondary to placental oxidative stress and inflammation, may lead to widespread maternal endothelial and metabolic dysfunction [26], but these factors are yet to be fully elucidated. Recent work has focused on the role of maternal plasma markers, implicating endothelial dysfunction in pre-eclampsia (e.g., sFlt-1, HIF-1 $\alpha$ and sENG), with expression abnormalities of these factors all noted in pre-eclampsia [27]. Furthermore, the contribution of associated maternal metabolic, inflammatory and coagulation parameter abnormalities have also been identified in this condition, but with no clear causal links to its development [27].

The diagnosis of pre-eclampsia is made clinically. However, recent assessment of biochemical markers and associated ultrasound features as diagnostic predictors of pre-eclampsia 
have been investigated. The use of biochemical markers to identify high-risk pregnancies would allow diagnostic ultrasound assessment at a relatively early stage, allowing early intervention prior to complications. Unfortunately, the markers so far investigated do not appear to have a high enough positive predictive value to use routinely in clinical practice [28]. A recent systematic review investigated levels of circulating PlGF, VEGF, sFlt1 and sEng in the serum and plasma of pregnant women prior to 30 weeks' gestation, but before clinical onset of pre-eclampsia [29]. Modest rises in PlGF, sFlt1 and sEng concentrations were all noted prior to 30 weeks' gestation in women who developed pre-eclampsia, but test accuracies for these markers remained too poor for accurate prediction of pre-eclampsia in clinical practice [29].

The use of first or second trimester maternal uterine artery Doppler velocimetry has been extensively investigated both in isolation and in combination with routinely measured first trimester serum markers (e.g., $\beta$-hCG, PAPP-A, inhibin $\mathrm{A}$ and activin $\mathrm{A}$ ) and maternal risk factor assessment [30]. A recent screening study for pre-eclampsia measured maternal central aortic systolic BP and arterial stiffness, as assessed by ultrasound pulse wave velocity and augmentation index in singleton pregnancies at $11+0$ to $13+6$ weeks' gestation and found that women who went on to develop pre-eclampsia had higher aortic systolic BP and arterial stiffness apparent from the first trimester of pregnancy [31]. One recent study using clinical, biochemical and ultrasound markers during the first trimester of pregnancy in 70 pregnant women who later developed pre-eclampsia and 289 control patients found that affected pregnancies were characterized by impaired placentation and an antiangiogenic state during the first trimester of pregnancy [32].

It seems likely that a multivariate screening model, as adopted worldwide for aneuploidy screening, will be the most successful in detecting early onset pre-eclampsia. However, an effective pre-eclampsia screening test is essentially meaningless if no effective treatment can be implemented that improves clinical outcome [33].

\section{Treatments}

Delivery of the placenta will resolve gestational hypertension and pre-eclampsia [1]. Therefore, a difficult choice often has to be made with regard to the correct time to intervene, so as to avoid both maternal systemic complications and neonatal iatrogenic prematurity. The shortterm treatment of chronic hypertension in pregnancy has its benefits in reducing rates of maternal cardiovascular morbidity and mortality [2]. However, as these women are treated for a $\leq 9$-month period, any short-term maternal health benefits should be balanced against possible shortand long-term consequences of antihypertensive therapy on fetal and neonatal health [21].

\section{Clinical monitoring}

Women with chronic hypertension, gestational hypertension and those at-risk of developing a hypertensive disorder of pregnancy should be managed by a consultant obstetrician conservatively until 34 weeks' gestation and closely monitored thereafter as close to term as possible (Figure 2) [102]. During this time, the consultant obstetrician with overall responsibility for care should document, on an individual basis, the threshold clinical and biochemical maternal and fetal values before 34 weeks' gestation prior to considering any intervention [102].

\section{Intrapartum care}

The birth, by either induction of labor or cesarean section, should be based upon the clinical circumstances and the woman's preference as far as possible. Delivery before 34 weeks' gestation should be offered if a woman develops severe refractory hypertension or documented threshold maternal intervention values are breached [102]. Delivery at these earlier gestations will often be by cesarean section as an induction process is more likely to fail due to poor cervical ripening in response to prostaglandin priming [34]. Birth should take place following anesthetic and neonatal consultation and should occur following maternal corticosteroid administration [102].

\section{Individual interventions}

\section{- Lifestyle}

The NICE guidelines on hypertension in pregnancy recommend that women at risk of hypertensive disorders should follow the same advice on rest, exercise and work as that for healthy pregnant women [102]. Clinicians should reassure women with hypertension that it is safe to continue working and to begin or continue a moderate course of exercise during pregnancy [102]. 
Diet

Salt restriction is not recommended to prevent gestational hypertension or pre-eclampsia. Nutritional supplementation (e.g., magnesium, folic acid, vitamins $\mathrm{C}$ and $\mathrm{E}$, fish oils, algal oils and garlic) is also not recommended for these purposes [102]. Low dietary calcium has been previously hypothesized to cause maternal hypertension, by causing negative feedback within the calcium homeostatic cycle, promoting increased vascular smooth muscle cell calcium and, thus, vasoconstriction. The most recent Cochrane systematic review analyzing several calcium supplementation trials in pregnancy found that calcium reduced the risk of high BP, particularly for women at

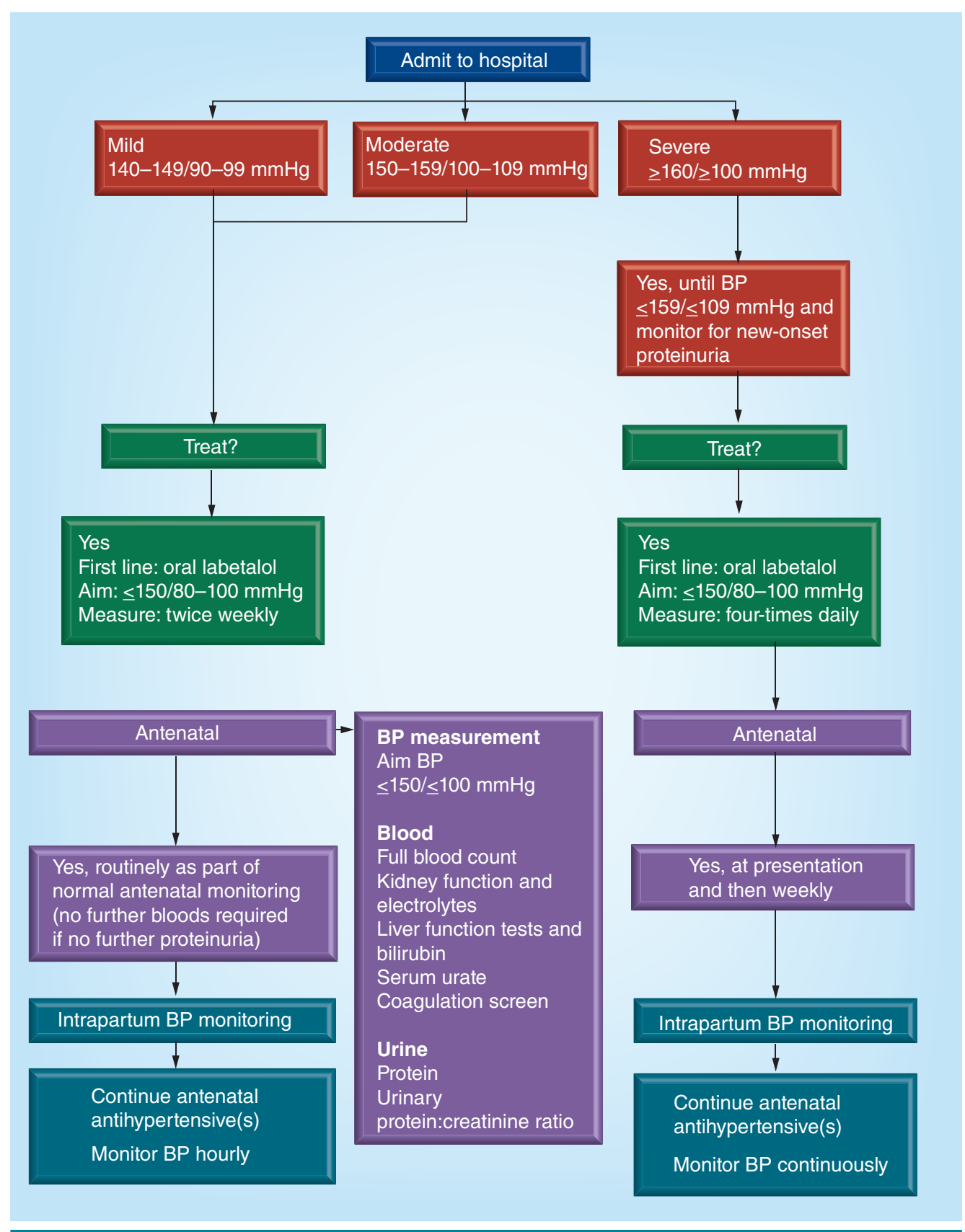

Figure 2. Management of hypertension in pregnancy.

BP: Blood pressure.

Adapted from [102]. 
high risk of pre-eclampsia, as well as reducing serious maternal morbidity and mortality (with no effect on preterm delivery rates or low infant birth weight) [35]. However, supplementation has not been shown to reduce hypertension/ pre-eclampsia incidence rates in women with a normal calcium intake and, therefore, this strategy has not yet been adopted at a population level.

The role of antioxidant supplementation for the prevention of pre-eclampsia was previously investigated by the VIP randomized control trial, which found that vitamins $\mathrm{C}$ and $\mathrm{E}$ supplementation did not prevent pre-eclampsia in women at risk, but did increase the rate of babies born with a low birth weight [36]. Therefore, the routine use of antioxidants during pregnancy for the prevention of preeclampsia and other outcomes is not supported [37]. However, a more recent study has found that maternal dietary supplementation with L-arginine and antioxidant vitamins together did reduce pre-eclampsia and eclampsia incidence rates in a high-risk cohort [38], indicating the need for further evaluation of these supplements combined in a low-risk population to determine the generalizability of this seemingly protective effect. However, the majority of the current, high-quality evidence for antioxidant use in pregnancy shows little or no benefit.

\section{Pharmacological therapies during pregnancy \\ - Antihypertensive therapies}

Conservative management of hypertension in pregnancy should be considered in all women, especially if presenting early in pregnancy, to reduce the risk of fetal hypoperfusion. Antihypertensive treatment is, of course, essential in severe hypertension to prevent maternal complications. A systolic BP $\geq 160 \mathrm{mmHg}$ or diastolic BP $\geq 110 \mathrm{mmHg}$ risks direct arterial damage, resulting in systemic complications such as maternal cerebral hemorrhage, cardiac failure and placental abruption [39]. A Cochrane systematic review of 46 studies trialing antihypertensives in mild/moderate severity hypertension during pregnancy $(n=3200$, comparing treatment vs placebo) found that the incidence of severe hypertension was halved by the use of antihypertensives versus placebo, although no effect was noted on the incidence of pre-eclampsia [39]. A comparison of antihypertensive agents were studied in the same Cochrane review $(n=1282)$, which found that $\beta$-blockers appeared to be better than methyldopa at reducing the risk of severe hypertension only [39].

As there is currently no consensus regarding management of mild/moderate hypertension in pregnancy, the results of an ongoing randomized controlled trial, the Control of Hypertension in Pregnancy study, is eagerly awaited in 2013 [103]. Pilot data from Control of Hypertension in Pregnancy study released in 2009 indicated an improved perinatal outcome in the less tightly controlled BP group, without a consequent increase in proteinuria or maternal pre-eclamptic end-organ complications [40]. However, these women developed severe hypertension more frequently [40], which may be associated with an increased risk of associated morbidities (e.g., hemorrhagic stroke).

The use of antihypertensives is split into first-, second- and third-line agents. First-line agents include methyldopa or an adrenoceptor antagonist (e.g., labetalol) [102]. Second-line agents include a vasodilator, often a calciumchannel antagonist (e.g., nifedipine or hydralazine) [102]. Third-line treatment is the additional use of either an adrenoceptor agonist or methyldopa [102]. As can be ascertained, there are few antihypertensive agents available that have been safely trialed in pregnancy. A starting regimen for maternal hypertension may include labetalol $200 \mathrm{mg}$ three-times daily, increasing to $300 \mathrm{mg}$ four-times daily if required [102]. If the BP remains uncontrolled, long-acting calcium channel antagonists (e.g., nifedipine LA $30-60 \mathrm{mg}$ once daily) may be added [102]. If the $\mathrm{BP}$ continues to be high on the maximum doses of a first- and second-line agent in combination, then a short trial of a third-line agent may be attempted prior to arranging, often early, delivery [102]. In this case, methyldopa $0.25 \mathrm{~g}$ two-/threetimes daily, increasing to $1.0 \mathrm{~g}$ three-times daily, may be advocated. Unlike in the nonpregnant population, angiotensin-converting (ACE) enzyme inhibitors and angiotensin II receptor antagonists (ARBs) are not used during pregnancy for maternal hypertension management due to known teratogenic and fetotoxic side effects [102]. These drugs should be discontinued prior to conception and an alternative agent started [102].

As there are clear distinctions between the use of antihypertensives in the pregnant and 
nonpregnant populations, the mode of action of several commonly used agents are detailed below.

\section{Methyldopa}

Methyldopa is the most extensively studied drug used in the pregnant population. It is a centrally acting $\alpha$-receptor blocker and is now the preferred agent, over $\beta$-blockers, to manage chronic hypertension in pregnancy [102]. Now rarely used in the nonpregnant population, its known safety profile means it remains a first-line agent, with evidence suggesting this drug has fewer direct effects on the fetal vasculature than other antihypertensive agents [41]. However, methyldopa is associated with frequent side effects, including: loss of energy, dizziness, fatigue, depression, headache, vomiting and palpitations, affecting $15 \%$ of women in one study (although only the first two symptoms were significantly different between the control and treatment arms) [42]. The development of hemolytic anemia (detected by positive Coombs test) and elevated liver transaminases were also noted side effects. However, a long-term follow-up longitudinal trial found no long-term adverse side effects in the offspring [43].

\section{Adrenoreceptor antagonists}

Of the wide range of adrenoreceptor antagonists that exist, only labetalol, atenolol and oxprenolol have been studied in pregnancy [44-46]. These agents are often preferred clinically, especially if therapy is required in the third trimester, having better side-effect profiles than methyldopa, as well as appearing to be better at reducing rates of severe maternal hypertension [41]. They are highly effective, although long-term use of these agents has been linked to fetal intrauterine growth restriction [47,48]. Labetalol has comparatively less impact on fetal growth [46] and is therefore more commonly used in antenatal management of hypertension, with the possibility of conversion to atenolol in the postnatal period [102]. The effect on fetal growth is likely due to the reduction in maternal BP causing a degree of fetal hypoperfusion, rather than a specific side effect of this class of antihypertensives [47]. Changing to atenolol postnatally has important concordance implications for the new mother due to this drug requiring only once-daily dosing [49] compared with multiple daily doses with labetalol [49], although this may be difficult to initiate and monitor following hospital discharge. In the short term, these agents appear safe for the growing fetus, although the same safety profile information does not yet exist for this antihypertensive class as it does for methyldopa.

\section{Calcium channel antagonists}

Calcium channel antagonists act by causing rapid-onset vasodilation by blocking calcium influx into smooth muscle cells and are delivered via oral or sublingual routes. They are extensively used in the nonpregnant population and have an established use in the pregnant population. The NICE guidance on these agents suggests that there is no evidence to justify the clinical effectiveness of these drugs [102]. However, in pregnant animal models, an infusion of this agent has been shown to reduce vascular sensitivity, and in human studies has been shown to reduce human maternal cerebral artery flow velocity, which may be important in reducing cerebral ischemia in pre-eclampsia [50]. They cause no known major maternal or fetal side effects, but are known to cause several other side effects, including tachycardia, facial flushing, warm, sweaty peripheries and headache. The use of nifidepine may also be contraindicated if the use of magnesium sulfate is warranted for the treatment of eclamptic seizures, due to a prolongation of nifidipine activity causing profound maternal hypotension [51].

\section{Hydralazine}

Hydralazine acts by inhibiting vascular smooth muscle cell contraction, although the precise mode of action is still unknown. It is primarily used in the acute setting to reduce severe hypertension and is delivered by intramuscular injection or intravenous bolus/infusion [52]. It acts within $20-30 \mathrm{~min}$, at which point it also induces a tachycardia (owing to its side effect of prolonging noradrenaline release, as well as the degree of hypotension achieved causing a baroreceptor-mediated reflex tachycardia) [52]. Side effects may include anxiety, restlessness and headache [52]. As these side effects may mimic the clinical presentation of severe pre-eclampsia, hydralazine is more commonly used as a secondline treatment in combination with another firstline agent, to help inhibit its sympathetic side 
effects [52]. Current evidence does not support hydralazine as a first-line, sole treatment for severe hypertension in pregnancy, owing to the increased rates of maternal and neonatal morbidity associated with its use [52].

\section{Thiazide diuretics}

Thiazide diuretics are used infrequently in pregnancy. These drugs increase the risk of congenital abnormalities and neonatal complications [53]. Therefore, women should discuss other antihypertensive treatments with their healthcare professional if they are planning a pregnancy [102].

\section{ACE inhibitors \& ARBs}

ACE inhibitors and ARBs should be avoided prior to conception and during pregnancy, owing to the increased risk of teratogenic and fetotoxic side effects $[54,55]$. However, it is likely that the elevated risks may not be specific to these drugs, but are instead related to maternal factors and diseases that typically coexist with hypertension in pregnancy, such as diabetes, advanced maternal age and obesity [56].

\section{Magnesium sulfate}

The Magpie study, published in 2002, investigated the use of magnesium sulfate in the prevention of pre-eclampsia in at-risk women. The study found that its use halved the incidence of eclampsia in at-risk women with pre-eclampsia [57]. Follow-up studies looking at 2-year maternal outcome [58] and 18-month offspring outcome [59] have so far found no evidence of increased risk of disability or death and, therefore, this agent can be safely used as an anticonvulsant for women with pre-eclampsia at risk of developing eclampsia.

\section{- Antithrombotic therapies Aspirin}

Low-dose aspirin prophylaxis has long been of interest because it is thought to correct an imbalance between the thromboxane $\mathrm{A}_{2}$ :prostacyclin ratio that is associated with increased vasoreactivity. Women with at least one high risk factor or at least two moderate risk factors for pre-eclampsia should take $75 \mathrm{mg}$ of aspirin daily from 12 weeks' gestation until the birth of the baby to prevent recurrence [60], although clinical decisions should be made on an individual basis.

\section{Low-molecular-weight heparin}

Pre-eclampsia is related to reduced uteroplacental blood flow and placental thrombotic and inflammatory lesions. Low-molecular-weight heparin acts as an anticoagulant by inactivating thrombin and other proteases involved in hemostasis. An improvement in pregnancy outcome has been noted in women with a history of severe pre-eclampsia if low-molecular-weight heparin was administered [6]. However, so far, routine use of low-molecular-weight heparin as a preventive agent has not been implemented in clinical practice.

\section{- Potential new therapies}

The role of one aberrant maternal antiangiogenesis marker in pre-eclampsia has also been recently exploited for therapeutic use. A preliminary study investigated the extracorporeal removal of sFlt-1 from maternal plasma in five women with very preterm pre-eclampsia [62]. It found that extracorporeal apheresis could lower the elevated circulating sFlt-1, leading to reduced proteinuria, stabilization of maternal BP, without apparent adverse events to the mother and fetus and prolongation of pregnancy in a treatment frequency-dependant fashion [62]. This offers a potential new treatment approach for the pre-eclampsia spectrum.

\section{Pharmacology after pregnancy}

As highlighted, maternal BP drops in the immediate postpartum period, before rising again during the first 5 days postdelivery [63]. Vigilance and adherence to an antihypertensive regimen and BP monitoring are extremely important during this time period. First, this should avoid precipitating maternal hypotension with aggressive use of antenatal hypertensive agents and, second, avoid false reassurance that the woman no longer has a hypertensive disorder. One recent Australian dataset examining maternal mortality found the majority of eclamptic seizures to occur during labor (44.1\%), with a substantial number (26.3\%) occurring during the postnatal period (median day of postnatal seizure occurrence was day 4 [range: 1-55]) [64]. Therefore, midwives following up these women in the community must be aware of any antenatal hypertensive condition and should make appropriate referrals to obstetric units should postnatal BP or symptoms of pre-eclampsia/eclampsia occur. 
Hypertensive control should be achieved prior to discharge and women with hypertension in pregnancy should have their BP measured daily for the first 2 days and at least once between days 3 and 5 postdelivery [102]. Should they require an antihypertensive at this stage, a $\beta$-blocker (e.g., atenolol or labetalol) is preferred to methyldopa due to a reported association with postpartum depression, although this is now thought to be much weaker than originally believed [65].

If a second agent is required, a calcium channel blocker or ACE inhibitor may be added. These medications can often be stopped 6 weeks postpartum, at which point women with gestational hypertension or pre-eclampsia should have returned to their prepregnancy BP values [102]. Women with chronic hypertension can then resume their prepregnancy antihypertensive regime [102]. Should a woman choose to breastfeed, she should be advised that there is insufficient evidence on the safety profile of ACE inhibitors (other than enalapril or captopril), ARBs or amlodipine and that these drugs should be avoided to prevent breast milk excretion [102].

Long-term management \& risk

stratification

- Future pregnancies

Women with chronic hypertension are advised to seek prepregnancy counseling either in primary care or from a multidisciplinary team including consultant obstetricians and hospital physicians [102]. The secondary care setting is particularly important if the woman has existing comorbidities (e.g., renal disease or diabetes), which have caused or exacerbated her hypertension. Antihypertensive medication should be reviewed so that any ACE inhibitor or ARB treatment prior to conception can be stopped and an alternative antihypertensive regime commenced. Associated cardioprotective agents, such as statins, should also be stopped prior to conception [102]. These women with chronic hypertension should then be referred to consultant-led antenatal care to have increased antenatal BP monitoring to detect superimposed pre-eclampsia [102].

If a woman develops gestational hypertension, she has an increased risk of between 16 and 47\% of developing it in a future pregnancy [102], and this should be considered in planning routine antenatal care. If pre-eclampsia occurs, there is a $16 \%$ increased risk of this condition affecting future pregnancies [102]. Owing to this increased risk, women who have developed pre-eclampsia in a prior pregnancy are advised to achieve and maintain a healthy BMI before any further pregnancies (although this should form part of prepregnancy advice for all women).

\section{- Long-term maternal cardiovascular complications}

There is increasing evidence to suggest that hypertension in pregnancy, in particular pre-eclampsia, is associated with developing cardiovascular disease in later life [66,67]. Severe maternal hypertension is associated with an increased risk of developing ischemic heart disease (IHD) [68]. One population study found that the risk of developing IHD was higher in women who developed pre-eclampsia or eclampsia in pregnancy than in women solely with hypertension [69]. Another study found that the combination of pre-eclampsia, preterm delivery and low birthweight was associated with a sevenfold increased risk of hospital admission or death secondary to IHD (relative risk: 7; 95\% CI: 3.3-14.5) [70]. This increased risk was also found within a meta-analysis that reported that a twofold increased risk of IHD in women who had developed pre-eclampsia remained after adjusting for several other maternal cardiovascular risk factors (e.g., pregestational hypertension, diabetes, obesity and smoking) [71]. In particular, the timing of pre-eclampsia occurrence was associated with differing IHD risks. When pre-eclampsia occurred before 37 weeks' gestation, women were found to have an eightfold increased risk of developing IHD [71]. In addition, pre-eclampsia has also been linked with a higher risk of developing end-stage renal disease [72]. Pregnancy itself appears to be associated with an increased risk of cardiovascular disease. This has been hypothesized to be due to the pregnancy state mimicking that of the metabolic syndrome and its adverse effects of relative insulin insensitivity and dyslipidemia. A theory explaining this association was proposed by Sattar and Greer, who suggest that women predisposed to developing a temporary metabolic syndrome during pregnancy may have an increased propensity of developing metabolic and cardiovascular disease in later life [73].

This increased risk of future cardiovascular disease associated with a history of preeclampsia 
Box 2. Hypertensive disorders of pregnancy: summary of best practices.

- Arterial hypertension in pregnancy is defined as diastolic BP $\geq 90 \mathrm{mmHg}$ on more than one occasion

$\geq 4$ h apart

- The main types are: chronic, gestational and pre-eclampsia (de novo or superimposed)

- Women with chronic hypertension should have optimal BP management (including appropriate antihypertensive selection) prior to conception

- Methyldopa is the first-line agent for chronic hypertension and labetalol should be first-line treatment for pre-eclampsia

- Magnesium sulfate can be used safely as an anticonvulsant for women with pre-eclampsia at risk of developing eclampsia

- BP vigilance and adherence to an antihypertensive regimen are extremely important in the postpartum period

- ACE inhibitors, ARBs and thiazide diuretics should be avoided during pregnancy and while breast feeding

- Clinicians should inform patients of their increased risk of cardiovascular and renal disease in later life ACE: Angiotensin-converting enzyme; ARB: Angiotensin II receptor antagonist; BP: Blood pressure.

has been recognized in the UK by the NICE Hypertension in Pregnancy guidelines, which advises all clinicians to inform their patients of these risks so as to undergo routine monitoring for the development of further hypertensive or cardiovascular disease [102]. These women should be encouraged, within primary care, to reduce their cardiovascular disease risk profile by maintaining a healthy weight through regular diet and exercise, and undergoing regular screening for hypertension, hyperlipidemia and diabetes [102]. The offspring of pre-eclampsiaaffected pregnancies also have an increased risk of higher BP with almost double the risk of stroke in later life [74]. There are therefore hypothetical potential effects of pre-eclampsia on future generations as these children go on to produce their own offspring.

\section{Conclusion \& future perspective}

Hypertension in pregnancy remains a key topic that all clinicians managing pregnant women should remain aware of in order to prevent substandard care leading to maternal and fetal/neonatal deaths (Box 2). At present, the recognition of hypertensive disorders remains part of the routine antenatal monitoring and screening in the UK. In the future, it is hoped that the development of accurate screening markers will allow the early recognition of these high-risk pregnancies, allowing care to focus specifically on those women likely to encounter complications.

A significant future development will be in the identification of the causative factor(s) implicated in pre-eclampsia, with future work focusing on the differentiation between early-and late-onset disease in terms of etiology and management. A successful screening tool is anticipated, likely to be in the form of a serum or urinary marker or combination of markers, prior to further monitoring investigations (e.g., Doppler ultrasound). Systematic reviews have concluded that no single test fulfilling WHO criteria for biomarker selection can currently diagnose/predict pre-eclampsia, but this does not mean that this cannot be achieved in the future. If identified, the pathological pathway behind the predictive marker(s) may also serve as a therapeutic target for future treatments.

The recognition of adverse pregnancy outcomes being associated with both maternal and offspring long-term cardiovascular disease risk [75,76] may indicate the future need to employ drugs that protect the system in pregnancy, especially as increasingly more cardiovascular disease is diagnosed in the adult female population. A screening program to identify those women (and, potentially, children) affected by adverse pregnancy outcomes would allow targeted therapies to help reduce the current burden of cardiovascular disease in a timely manner.

Financial \& competing interests disclosure

The authors have no relevant affiliations or financial involvement with any organization or entity with a financial interest in or financial conflict with the subject matter or materials discussed in the manuscript. This includes employment, consultancies, honoraria, stock ownership or options, expert testimony, grants or patents received or pending, or royalties.

No writing assistance was utilized in the production of this manuscript. 


\section{References}

Papers of special note have been highlighted as:

- of interest

-1 of considerable interest

1 Visintin C, Mugglestone MA, Almerie MQ et al. Management of hypertensive disorders during pregnancy: summary of NICE guidance. BMJ 25, 341c2207 (2010).

2 Bhutta ZA, Cabral S, Chan CW et al. Reducing maternal, newborn, and infant mortality globally: an integrated action agenda. Int. J. Gynaecol. Obstet. 119(Suppl. 1), S13-S17 (2012).

3 Cantwell R, Clutton-Brock T, Cooper G; Centre for Maternal and Child Enquiries (CMACE). Saving mothers' lives: reviewing maternal deaths to make motherhood safer: 2006-08. The Eighth Report on Confidential Enquiries into Maternal Deaths in the United Kingdom. BJOG 118(Suppl. 1), S1-S203 (2011).

-n An accurate, up-to-date reflection of the impact of hypertensive disease on the maternal and neonatal population in the UK.

4 Mahendru AA, Everett TR, Wilkinson IB, Lees CC, McEniery CM. Maternal cardiovascular changes from pre-pregnancy to very early pregnancy. J. Hypertens. 30(11), 2168-2172 (2012).

5 Grindheim G, Estensen ME, Langesaeter E, Rosseland LA, Toska K. Changes in blood pressure during healthy pregnancy: a longitudinal cohort study. J. Hypertens. 30(2), 342-350 (2012).

6 Gaillard R, Bakker R, Willemsen SP, Hofman A, Steegers EA, Jaddoe VW. Blood pressure tracking during pregnancy and the risk of gestational hypertensive disorders: the Generation R Study. Eur. Heart J. 32(24), 3088-3097 (2011).

7 Chung JG, Taylor RS, Thompson JM et al. Gestational weight gain and adverse pregnancy outcomes in a nulliparous cohort. Eur. J. Obstet. Gynecol. Reprod. Biol. 167(2), 149-153 (2013).

8 Robson SC, Boys RJ, Hunter S, Dunlop W. Maternal hemodynamics after normal delivery and delivery complicated by postpartum hemorrhage. Obstet. Gynecol. 74(2), 234-239 (1989).

9 Shennan A, Gupta M, Halligan A, Taylor DJ, de Swiet M. Lack of reproducibility in pregnancy of Korotkoff Phase IV as measured by mercury sphygmomanometry. Lancet 347(8995), 139-142 (1996).

10 Brown MA, Reiter L, Smith B, Buddle ML, Morris R, Whitworth JA. Measuring blood pressure in pregnant women: a comparison of direct and indirect methods. Am. J. Obstet. Gynecol. 171(3), 661-667 (1994).

11 Natarajan P, Shennan AH, Penny J et al. Comparison of auscultatory and oscillometric automated blood pressure monitors in the setting of preeclampsia. Am. J. Obstet. Gynecol. 181(5 Pt 1), 1203-1210 (1999).

12 Peek M, Shennan A, Halligan A et al. Hypertension in pregnancy: which method of blood pressure measurement is most predictive of outcome? Obstet. Gynecol. 88(6), 1030-1033 (1996).

13 Brown MA, Lindheimer MD, de Swiet M, Van Assche A, Moutquin JM. The classification and diagnosis of the hypertensive disorders of pregnancy: statement from the International Society for the study of Hypertension in Pregnancy (ISSHP). Hypertens. Pregnancy 20(1), 9-14 (2001).

14 Martin JN Jr, Thigpen BD, Moore RC et al. Stroke and severe preeclampsia and eclampsia: a paradigm shift focusing on systolic blood pressure. Obstet. Gynecol. 105(2), 246-254 (2005).

15 Lindenstrøm E, Boysen G, Nyboe J. Influence of systolic and diastolic blood pressure on stroke risk: a prospective observational study. Am. J. Epidemiol. 142(12), 1279-1290 (1995).

16 Macdonald-Wallis C, Lawlor DA, Fraser A et al. Blood pressure change in normotensive, gestational hypertensive, preeclamptic, and essential hypertensive pregnancies. Hypertension 59(6), 1241-1248 (2012).

- Retrospective analysis of a cohort study identifying characteristic changes in maternal hypertension readings across gestation.

17 Brown MA, Hague WM, Higgins J et al. Austalasian Society of the study of hypertension in pregnancy. The detection, investigation and management of hypertension in pregnancy: full consensus statement. Aust. NZ J. Obstet. Gynaecol. 40(2), 139-155 (2000).

18 O'Brien TE, Ray JG, Chan WS. Maternal body mass index and the risk of preeclampsia: a systematic overview. Epidemiology 14(3), 368-374 (2003).

19 Sibai BM. Chronic hypertension in pregnancy. Obstet. Gynecol. 100(2), 369-377 (2002).

20 Ames M, Rueda J, Caughey AB. Ambulatory management of chronic hypertension in pregnancy. Clin. Obstet. Gynecol. 55(3), 744-755 (2012).
21 Chappell LC, Enye S, Seed P et al. Adverse perinatal outcomes and risk factors for preeclampsia in women with chronic hypertension: a prospective study. Hypertension 51(4), 1002-1009 (2008).

22 Kamoun M, Mnif MF, Charfi $\mathrm{N}$ et al. Adrenal diseases during pregnancy: pathophysiology, diagnosis and management strategies. Am. J. Med. Sci. (2013) (Epub ahead of print).

23 Steegers EA, von Dadelszen P, Duvekot JJ, Pijnenborg R. Pre-eclampsia. Lancet 376, 631-644 (2010).

24 Ghulmiyyah L, Sibai B. Maternal mortality from preeclampsia/eclampsia. Semin. Perinatol. 36(1), 56-59 (2012).

25 Mor G, Cardenas I, Abrahams V, Guller S. Inflammation and pregnancy: the role of the immune system at the implantation site. Ann. NY Acad. Sci. 1221, 80-87 (2011).

26 Redman CW, Sargent IL. Placental stress and pre-eclampsia: a revised view. Placenta 30(Suppl. A), S38-S42 (2009).

27 Grill S, Rusterholz C, Zanetti-Dällenbach R et al. Potential markers of preeclampsia - a review. Reprod. Biol. Endocrinol. 7, 70 (2009).

28 Kuc S, Wortelboer EJ, van Rijn BB et al. Evaluation of 7 serum biomarkers and uterine artery Doppler ultrasound for first-trimester prediction of preeclampsia: a systematic review. Obstet. Gynecol. Surv. 66(4), 225-239 (2009).

29 Kleinrouweler CE, Wiegerinck MM, Ris-Stalpers C et al. Accuracy of circulating placental growth factor, vascular endothelial growth factor, soluble fms-like tyrosine kinase 1 and soluble endoglin in the prediction of pre-eclampsia: a systematic review and meta-analysis. BJOG 119(7), 778-787 (2012).

30 Jodicke C, Maulik D, Singh P, Heitmann E, Maulik D. Role of ultrasound in preeclampsia. Minerva Ginecol. 64(4), 293-308 (2012).

31 Khalil A, Akolekar R, Syngelaki A, Elkhouli $\mathrm{M}$, Nicolaides K. Maternal hemodynamics at 11-13 weeks' gestation and risk of preeclampsia. Ultrasound Obstet. Gynecol. 40(1), 28-34 (2012).

32 Parra-Cordero M, Rodrigo R, Barja P et al. Prediction of early and late pre-eclampsia from maternal characteristics, uterine artery Doppler and markers of vasculogenesis during the first trimester of pregnancy. Ultrasound Obstet. Gynecol. 41(5), 538-544 (2012).

33 Smith GC. Researching new methods of screening for adverse pregnancy outcome: 
lessons from pre-eclampsia. PLoS Med. 9(7), e1001274 (2012).

- Balanced, expert review on the current methods of screening for adverse pregnancy outcome.

34 Timmons B, Akins M, Mahendroo M. Cervical remodeling during pregnancy and parturition. Trends Endocrinol. Metab. 21(6), 353-361 (2010).

35 Buppasiri P, Lumbiganon P, Thinkhamrop J et al. Calcium supplementation (other than for preventing or treating hypertension) for improving pregnancy and infant outcomes. Cochrane Database Syst. Rev. 10, CD007079 (2011).

36 Poston L, Briley AL, Seed PT et al. Vitamins in Pre-eclampsia (VIP) Trial Consortium.

Vitamin $\mathrm{C}$ and vitamin $\mathrm{E}$ in pregnant women at risk for pre-eclampsia (VIP trial), randomised placebo-controlled trial. Lancet 367(9517), 1145-1154 (2006).

37 Rumbold A, Duley L, Crowther CA et al. Antioxidants for preventing pre-eclampsia. Cochrane Database Syst. Rev. 23(1), CD004227 (2008).

38 Vadillo-Ortega F, Perichart-Perera O, Espino $S$ et al. Effect of supplementation during pregnancy with L-arginine and antioxidant vitamins in medical food on pre-eclampsia in high risk population: randomised controlled trial. BMJ342, d2901 (2011).

39 Abalos E, Duley L, Steyn DW et al. Antihypertensive drug therapy for mild to moderate hypertension during pregnancy. Cochrane Database Syst. Rev. 24(1), CD002252 (2007).

40 Magee LA, Abalos E, von Dadelszen P, Sibai B, Walkinshaw SA, CHIPS Study Group. Control of hypertension in pregnancy. Curr. Hypertens. Rep. 11(6), 429-436 (2009).

41 Houlihan DD, Dennedy MC, Ravikumar N et al. Anti-hypertensive therapy and the fetoplacental circulation: effects on umbilical artery resistance. J. Perinat. Med. 32(4), 315-319 (2004).

42 Redman CW, Beilin LJ, Bonnar J. Treatment of hypertension in pregnancy with methyldopa: blood pressure control and side effects. Br. J. Obstet. Gynaecol. 84(6), 419-426 (1977).

43 Cockburn J, Moar VA, Ounsted M, Redman CW. Final report of study on hypertension during pregnancy: the effects of specific treatment on the growth and development of the children. Lancet 1(8273), 647-649 (1982).

44 Pickles CJ, Symonds EM, Broughton Pipkin F. The fetal outcome in a randomized double- blind controlled trial of labetalol versus placebo in pregnancy-induced hypertension. Br. J. Obstet. Gynaecol. 96(1), 38-43 (1989).

45 Rubin PC, Butters L, Clark DM et al. Placebo-controlled trial of atenolol in treatment of pregnancy-associated hypertension. Lancet 1(8322), 431-434 (1983).

46 Gallery ED, Ross MR, Gyory AZ. Antihypertensive treatment in pregnancy: analysis of different responses to oxprenolol and methyldopa. BMJ 291(6495), 563-566 (1985).

47 Butters L, Kennedy S, Rubin PC. Atenolol in essential hypertension during pregnancy. $B M J$ 301(6752), 587-589 (1990).

48 Sibai BM, Gonzalez AR, Mabie WC, Moretti M. A comparison of labetalol plus hospitalization versus hospitalization alone in the management of preeclampsia remote from term. Obstet. Gynecol. 70(3 Pt 1), 323-327 (1987).

49 Podymow T, August P. Hypertension in pregnancy. Adv. Chronic Kidney Dis. 14(2), 178-190 (2007).

50 Serra-Serra V, Kyle PM, Chandran R et al. The effect of nifedipine and methyldopa on maternal cerebral circulation. Br. J. Obstet. Gynaecol. 104(5), 532-537 (1997).

51 Gonen R. Contemporary usage of obstetric magnesium sulfate: indication, contraindication, and relevance of dose. Obstet. Gynecol. 115(1), 186 (2010).

52 Magee LA, Cham C, Waterman EJ et al. Hydralazine for treatment of severe hypertension in pregnancy: meta-analysis. BMJ 327(7421), 955-960 (2003).

53 Newstead-Angel J, Gibson PS. Cardiac drug use in pregnancy: safety, effectiveness and obstetric implications. Expert Rev. Cardiovasc. Ther. 7(12), 1569-1580 (2009).

54 Cooper WO, Hernandez-Diaz S, Arbogast PG et al. Major congenital malformations after first-trimester exposure to ACE inhibitors. N. Engl. J. Med. 354(23), 2443-2451 (2006).

55 Chung NA, Lip GY, Beevers M, Beevers DG. Angiotensin-II-receptor inhibitors in pregnancy. Lancet 357(9268), 1620-1621 (2001).

56 Polifka JE. Is there an embryopathy associated with first-trimester exposure to angiotensin-converting enzyme inhibitors and angiotensin receptor antagonists? A critical review of the evidence. Birth Defects Res. A Clin. Mol. Teratol. 94(8), 576-598 (2012).

57 Altman D, Carroli G, Duley L et al. Do women with pre-eclampsia, and their babies, benefit from magnesium sulphate? The Magpie trial: a randomised placebocontrolled trial. Lancet 359(9321), 1877-1890 (2002).

58 Magpie Trial Follow-Up study Collaborative Group. The Magpie trial: a randomised trial comparing magnesium sulphate with placebo for pre-eclampsia. Outcome for women at 2 years. BJOG 114(3), 300-309 (2007).

59 Magpie Trial Follow-Up Study Collaborative Group. The Magpie trial: a randomised trial comparing magnesium sulphate with placebo for pre-eclampsia. Outcome for children at 18 months. BJOG 114(3), 289-299 (2007).

60 Duley L, Henderson-Smart DJ, Meher S, King JF. Antiplatelet agents for preventing pre-eclampsia and its complications. Cochrane Database Syst. Rev. 2, CD004659 (2007).

61 Rey E, Garneau P, David M et al. Dalteparin for the prevention of recurrence of placentalmediated complications of pregnancy in women without thrombophilia: a pilot randomized controlled trial. J. Thromb. Haemost. 7(1), 58-64 (2009).

62 Thadhani R, Kisner T, Hagmann $\mathrm{H}$ et al. Pilot study of extracorporeal removal of soluble fms-like tyrosine kinase 1 in preeclampsia. Circulation. 23, 124(8), 940-950 (2011).

63 Magee L, Sadeghi S. Prevention and treatment of postpartum hypertension. Cochrane Database Syst. Rev. 25(1), CD004351 (2005).

64 Thornton C, Dahlen H, Korda A, Hennessy A. The incidence of preeclampsia and eclampsia and associated maternal mortality in Australia from population-linked datasets: 2000-2008. Am. J. Obstet. Gynecol. doi: 10.1016/j.ajog.2013.02.042 (2013) (Epub ahead of print).

65 Huffman JC, Stern TA. Neuropsychiatric consequences of cardiovascular medications. Dialogues Clin. Neurosci. 9(1), 29-45 (2007).

66 Ray JG, Vermeulen MJ, Schull MJ, Redelmeier DA. Cardiovascular health after maternal placental syndromes (CHAMPS), population-based retrospective cohort study. Lancet 366(9499), 1797-1803 (2005).

67 Hastie CE, Smith GC, Mackay DF, Pell JP. Maternal risk of ischaemic heart disease following elective and spontaneous pre-term delivery: retrospective cohort study of 750 350 singleton pregnancies. Int. J. Epidemiol. 40(4), 914-919 (2011).

68 Wikström AK, Haglund B, Olovsson M, Lindeberg SN. The risk of maternal ischaemic heart disease after gestational hypertensive disease. BJOG 112(11), 1486-1491 (2005). 
69 Jónsdóttir LS, Arngrímsson R, Geirsson RT, Sigvaldason H, Sigfusson N. Death rates from ischemic heart disease in women with a history of hypertension in pregnancy. Acta Obstet. Gynecol. Scand. 74(10), 772-776 (1995).

70 Smith GC, Pell JP, Walsh D. Pregnancy complications and maternal risk of ischaemic heart disease: a retrospective cohort study of 129,290 births. Lancet 357(9273), 2002-2006 (2001).

71 Bellamy L, Casas JP, Hingorani AD, Williams DJ. Pre-eclampsia and risk of cardiovascular disease and cancer in later life: systematic review and meta-analysis. $B M J$ 335(7627), 974 (2007).

72 Davison JM, Lindheimer MD. Pregnancy and chronic kidney disease. Semin. Nephrol. 31(1), 86-99 (2011).
73 Sattar N, Greer IA. Pregnancy complications and maternal cardiovascular risk: opportunities for intervention and screening? BMJ 325(7356), 157-160 (2002).

-1 Details the novel hypothesis of a link between pregnancy complications and later-life maternal cardiovascular and metabolic disease.

74 Davis EF, Newton L, Lewandowski AJ et al. Pre-eclampsia and offspring cardiovascular health: mechanistic insights from experimental studies. Clin. Sci. (Lond.) 123(2), 53-72 (2012).

75 Forest JC, Charland M, Massé J et al. Candidate biochemical markers for screening of pre-eclampsia in early pregnancy. Clin. Chem. Lab Med. 50(6), 973-984 (2012).

76 Siddiqui N, Hladunewich M. Understanding the link between the placenta and future cardiovascular disease. Trends Cardiovasc. Med. 21(7), 188-193 (2011).

\section{- Websites}

101 WHO. Women's health. November 2009. www.who.int/mediacentre/factsheets/fs334/ en/index.html

102 National Collaborating Centre for Women's and Children's Health Commissioned by NICE. Hypertension in pregnancy: the management of hypertensive disorders during pregnancy. RCOG Press, London, UK (2011). www.nice.org.uk/nicemedia/ live/13098/50475/50475.pdf

103 The CHIPS trial (control of hypertension in pregnancy study). http://clinicaltrials.gov/ct2/show/ NCT01192412 